\title{
Explosion of limit cycles and chaotic waves in a simple nonlinear chemical system
}

\author{
Brøns, Morten; Sturis, Jeppe
}

Published in:

Physical Review E. Statistical, Nonlinear, and Soft Matter Physics

Link to article, DOI:

10.1103/PhysRevE.64.026209

Publication date:

2001

Document Version

Publisher's PDF, also known as Version of record

Link back to DTU Orbit

\section{Citation $(A P A)$ :}

Brøns, M., \& Sturis, J. (2001). Explosion of limit cycles and chaotic waves in a simple nonlinear chemical system. Physical Review E. Statistical, Nonlinear, and Soft Matter Physics, 64(2), 026209.

https://doi.org/10.1103/PhysRevE.64.026209

\section{General rights}

Copyright and moral rights for the publications made accessible in the public portal are retained by the authors and/or other copyright owners and it is a condition of accessing publications that users recognise and abide by the legal requirements associated with these rights.

- Users may download and print one copy of any publication from the public portal for the purpose of private study or research.

- You may not further distribute the material or use it for any profit-making activity or commercial gain

- You may freely distribute the URL identifying the publication in the public portal 


\title{
Explosion of limit cycles and chaotic waves in a simple nonlinear chemical system
}

\author{
Morten Brøns* \\ Department of Mathematics, Technical University of Denmark, DK-2800 Kongens Lyngby, Denmark \\ Jeppe Sturis ${ }^{\dagger}$ \\ Pharmacological Research 1, Novo Nordisk, DK-2880 Bagsvard, Denmark
}

(Received 14 January 2001; published 18 July 2001)

\begin{abstract}
We consider a simple model of an autocatalytic chemical reaction where a limit cycle rapidly increases to infinite period and amplitude, and disappears under variation of a parameter. We show that this bifurcation can be understood from seeing the system as a singular perturbation problem, and we find the bifurcation point by an asymptotic analysis. Scaling laws for period and amplitude are derived. The unphysical bifurcation to infinity disappears under generic modifications of the model, and for a simple example we show is replaced by a canard explosion, that is, a narrow parameter interval with an explosive growth of the amplitude. The bifurcation to infinity introduces a strong sensitivity that may result in chaotic dynamics if diffusion is added. We show that this behavior persists even if the kinetics is modified to preclude the bifurcation to infinity.
\end{abstract}

DOI: 10.1103/PhysRevE.64.026209

PACS number(s): 82.40.Bj, 89.75.Kd, 82.20.-w

\section{INTRODUCTION}

Reaction schemes based on the cubic autocatalator as defined in Eq. (1) below have been widely used as prototypes for investigations into nonlinear dynamics of chemical reactions. The central part of the scheme is a cubic autocatalytic step and a first-order decay of the catalyst,

$$
A+2 B \rightarrow 3 B \quad\left(\text { with rate } k_{1} a b^{2}\right), \quad B \rightarrow C \quad \text { (with rate } k_{2} b \text { ), }
$$

where $a, b$ are the concentrations of the reactants $A, B$ and $k_{1}, k_{2}$ are the rate constants. Here we are interested in studying reactions in closed systems where the reactant $A$ is produced by a slow first-order decay of a precursor $P$,

$$
P \rightarrow A \quad\left(\text { rate } k_{0} p\right) .
$$

Clearly, the reaction based on the schemes (1) and (2) will eventually stop as $P$ is consumed. However, if the reactant $P$ is initially present in a large amount, it is customary to invoke the pooled chemical approximation and assume that $P$ retains its initial concentration $p_{0}$ throughout the reaction. Introducing dimensionless variables, the differential equations for the reaction scheme assuming the reactor is well stirred become

$$
\frac{d u}{d t}=\mu-u v^{2}, \quad \frac{d v}{d t}=u v^{2}-v,
$$

where $u=\sqrt{k_{1} / k_{2}} a, v=\sqrt{k_{1} / k_{2}} b, t$ is the dimensionless time scaled from physical time by $k_{1}$ and $\mu$ $=\sqrt{k_{1} / k_{2}} k_{0} p_{0} / k_{2}$. This system allows limit cycles, corresponding to an oscillating reaction, for certain values of $\mu$. The oscillations are, of course, artefacts from the pooled approximation, but even if the slow consumption of $P$ is in-

\footnotetext{
*Electronic address: m.brons@mat.dtu.dk

${ }^{\dagger}$ Electronic address: jstu@novo.dk
}

cluded in a model the transient behavior will for a long time resemble the dynamics of the system (3) closely.

The system (3) has been studied in detail by Merkin, Needham, and Scott [1]. It was shown that the limit cycles rapidly increase to infinite amplitude and period as $\mu$ approaches a critical value $\mu_{\infty}$ from above. Clearly, this unphysical bifurcation to infinity limits the applicability of the model (3), and it becomes interesting to establish whether the reaction scheme can be modified to avoid this bifurcation while still retaining the simplicity of the pooled approximation. To this end, Merkin, Needham, and Scott [2] added the uncatalyzed reaction

$$
A \rightarrow B \quad\left(\text { rate } k_{3} a\right)
$$

yielding the differential equations

$$
\frac{d u}{d t}=\mu-u v^{2}-r u, \quad \frac{d v}{d t}=u v^{2}-v+r u,
$$

with $r=k_{3} / k_{2}$. It was shown that, as long as $r \neq 0$, an interval close to $\mu_{\infty}$ with rapid growth of amplitude and period persisted. However, the bifurcation to infinity did not occur as the amplitude of the limit cycles remains bounded. Gray, Roberts, and Merkin [3] further added a quadratic autocatalytic reaction,

$$
A+B \rightarrow 2 B \quad\left(\text { rate } k_{4} a b\right)
$$

resulting in

$$
\frac{d u}{d t}=\mu-u v^{2}-r u-s u v, \quad \frac{d v}{d t}=u v^{2}-v+r u+s u v,
$$

with $s=k_{4} / \sqrt{k_{1} k_{2}}$ and again obtained bounded trajectories only.

The rapid but bounded growth of limit cycles over a short parameter interval as it occurs in the systems (5) and (7) is 
(a)
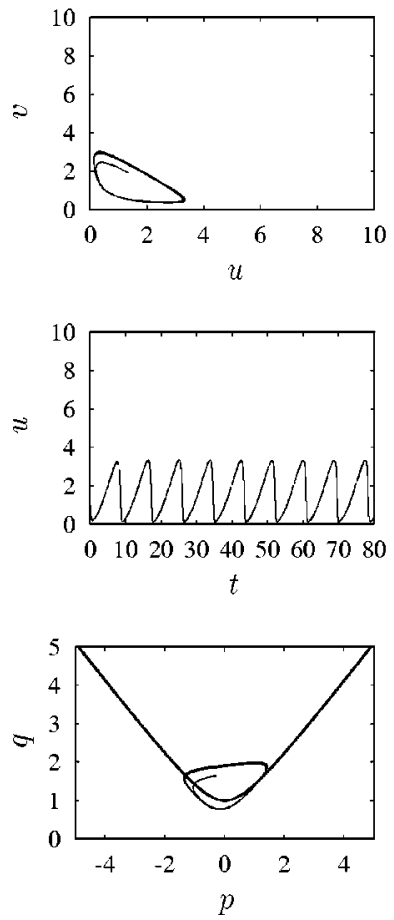

(b)
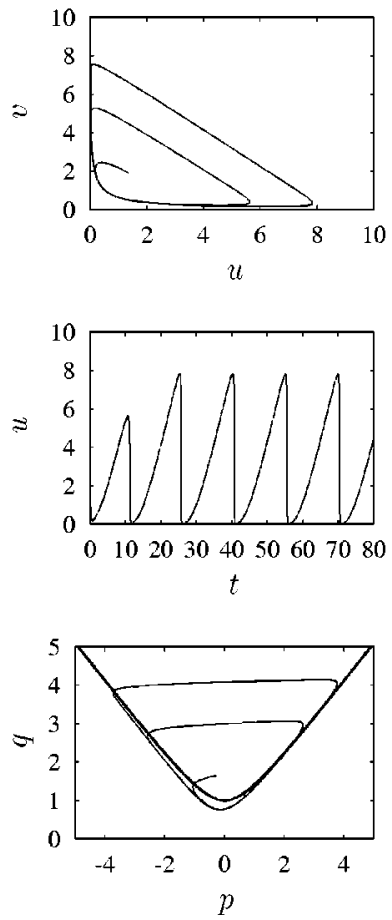

(c)
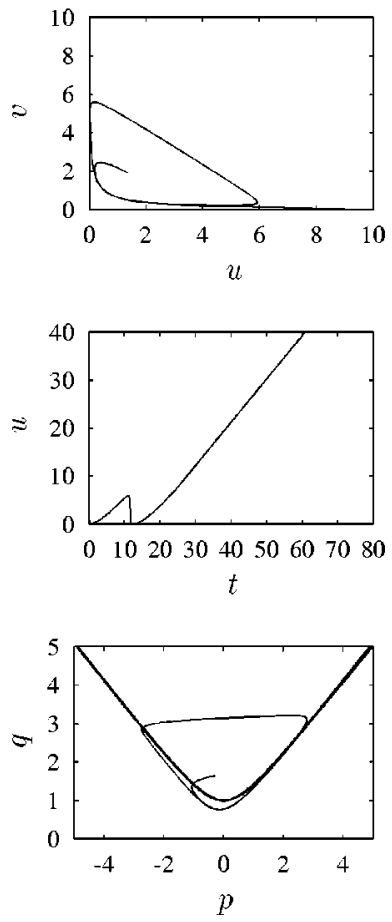

FIG. 1. Explosion and disappearance of the limit cycle of Eq. (3) for decreasing values of $\mu$. (a) $\mu=0.92$. (b) $\mu=0.9005$. (c) $\mu$ $=0.9$. Top row: Trajectories in the $(u, v)$ phase plane. Middle row: Time trace of $u$. Bottom row: Trajectories in the $(p, q)$ phase plane, defined by the coordinate transformation (16). Also shown is the slow manifold $S_{2}$ from (18). known as a canard explosion. This is mathematically well understood in singular perturbation systems of the form

$$
\frac{d u}{d t}=f(u, v, \mu, \epsilon), \quad \frac{d v}{d t}=\epsilon g(u, v, \mu, \epsilon)
$$

in the limit $\epsilon \rightarrow 0$ [4]. Canard explosions in chemical systems have been identified in the Oregonator [5] and the EdblomOrbán-Epstein (EOE) reaction [6].

In the present paper we show that it is possible to rewrite the system (3) as a singular perturbation system. This point of view gives rise to a simple geometric interpretation of the bifurcation to infinity and allows an asymptotic determination of $\mu_{\infty}$. We also obtain scaling laws for amplitude and period of the limit cycle as the bifurcation to infinity is approached that deviate from the results in [1], and we confirm our results by numerical computations. The singular perturbation analysis is basically the same as is applied in the analysis of the canard explosion. We also analyze the modi-

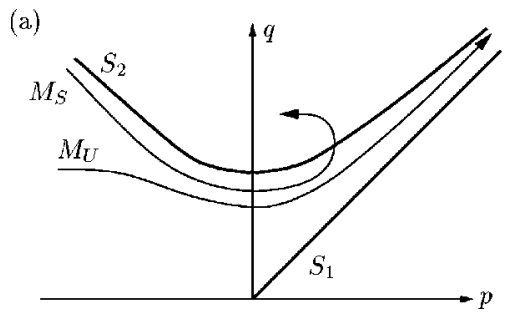

fied system (5) and give a simple geometric explanation why modifications of the original system (3), such as Eqs. (5) and (7), behave as they do.

We have previously $[7,8]$ analyzed bifurcations to infinity in models from mathematical economics and biochemistry. In those studies we have, as in [1], introduced critical points at infinity and seen the bifurcation as being associated with heteroclinic connections of the separatrices of these critical points. The present analysis is simpler in that it only involves objects in the Euclidean plane.

If the reactor is not well mixed, diffusion must be taken in account. If the reactor is spatially one dimensional the pooled kinetics of Eq. (3) leads to the reaction-diffusion system

$$
\frac{\partial u}{\partial t}=\lambda_{u} \frac{\partial^{2} u}{\partial x^{2}}+\mu-u v^{2}, \quad \frac{\partial v}{\partial t}=\lambda_{v} \frac{\partial^{2} v}{\partial x^{2}}+u v^{2}-v,
$$

where $\lambda_{v}, \lambda_{v}$ are diffusion coefficients. The basic pattern formation from local bifurcations subject to the no-flux boundary conditions

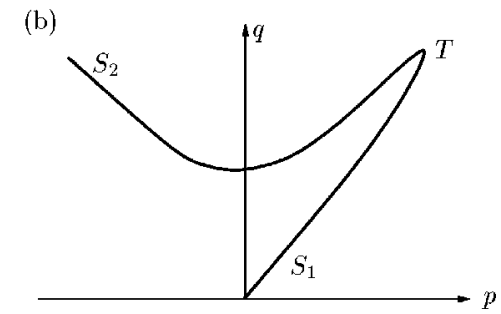

FIG. 2. Sketches of the $p$ and $q$ phase plane. (a) For the system (3), the branches $S_{1}$ and $S_{2}$ of the slow manifold with stable manifold $M_{S}$ and the unstable manifold $M_{U}$, both in the boundary layer of $S_{2}$. The configuration shown with $M_{S}$ above $M_{U}$ allows a limit cycle. (b) The slow manifold for the system (5) with turning point $T$ where the branches $S_{1}$ and $S_{2}$ meet. 
TABLE I. Results of the asymptotic calculation of $\mu_{\infty}$ for $\epsilon$ $=1$.

\begin{tabular}{cccc}
\hline \hline $\begin{array}{c}\text { Order } \\
k\end{array}$ & $\begin{array}{c}\text { Contribution } \\
\mu_{k}\end{array}$ & $\begin{array}{c}\text { Approximation } \\
\mu_{\infty}^{(k)}=\sum_{n=0}^{k} \mu_{n}\end{array}$ & $\begin{array}{c}\text { Relative error } \\
\left|\mu_{\infty}^{(k)}-\mu_{\infty}\right| / \mu_{\infty}\end{array}$ \\
\hline 0 & 1.00000000 & 1.00000000 & 0.11072139 \\
1 & -0.12500000 & 0.87500000 & 0.02811877 \\
2 & 0.03125000 & 0.90625000 & 0.00659126 \\
3 & -0.00488281 & 0.90136718 & 0.00116781 \\
4 & -0.00449970 & 0.89666748 & 0.00405224 \\
5 & 0.0757980 & 0.90424728 & 0.00436680 \\
6 & -0.00622034 & 0.89802694 & 0.00254226 \\
7 & 0.00072032 & 0.89874726 & 0.00174218 \\
8 & 0.00958743 & 0.90833470 & 0.00890678 \\
9 & -0.02425103 & 0.88408367 & 0.01802935 \\
10 & 0.03671743 & 0.092080109 & 0.02275347 \\
\hline \hline
\end{tabular}

$$
\frac{\partial u}{\partial x}=\frac{\partial v}{\partial x}=0 \text { for } x=0,1
$$

has been studied in some detail $[9,10]$. In $[11]$ it was noted that the amplitude of limit cycles born in a Hopf bifurcation may obtain large amplitudes as $\mu$ is decreased. We analyze this behavior for a case where both the diffusion constants are small and show that the bifurcation to infinity introduces a sensitivity that may result in chaotic dynamics. Finally, we show that this behavior is basically linked to the singular perturbation nature of the problem, as it persists even if the kinetics is modified to the form (5).

\section{DYNAMICS OF THE WELL-STIRRED REACTOR}

\section{A. Singular perturbation of the pooled model}

The system (3) has a critical point $(u, v)=(1 / \mu, \mu)$ which is stable for $\mu>1$ and loses stability in a Hopf bifurcation at $\mu=1$. By standard procedures [1] one finds the bifurcation to be supercritical, so stable limit cycles exist for $\mu<1$. As $\mu$ is decreased the amplitude and period grows rapidly, and when $\mu$ is decreased beyond the numerically obtained value

$$
\mu_{\infty}=0.9003157807722 \text {; }
$$

the limit cycle disappears and only unbounded trajectories remain. See Fig. 1.

The limit cycles display the characterstics of relaxation oscillations: a slow phase is followed by fast, almost linear motion. A formulation as a singular perturbation problem can be achieved by following the analysis of [6] for the EOE reaction. The key is the fact that the right hand sides of the differential equations are almost identical, except for a sign. Indeed, the system can be written in the form

$$
\frac{d u}{d t}=f(u, v)+g(u, v, \mu), \quad \frac{d v}{d t}=-f(u, v),
$$

with

$$
f(u, v)=v-u v^{2}, \quad g(u, v, \mu)=\mu-v .
$$

If $g$ is replaced by 0 in Eq. (12) the isolated critical point is replaced by two curves of critical points,

$$
v=0 \text { and } 1-u v=0 \text {. }
$$

Hence, if we modify (12) to include an auxiliary parameter $\epsilon$ as

$$
\frac{d u}{d t}=f(u, v)+\epsilon g(u, v, \mu), \quad \frac{d v}{d t}=-f(u, v),
$$

we obtain a singular perturbation problem in the limit $\epsilon=0$, and we recover the original problem when $\epsilon=1$. With the change of variables

$$
p=\frac{u-v}{2}, \quad q=\frac{u+v}{2}
$$

we obtain the system in the standard singular perturbation form,

$$
\frac{d p}{d t}=f(q+p, q-p), \quad \frac{d q}{d t}=\epsilon \frac{g(q+p, q-p, \mu)}{2} .
$$

The curves of equilibria (14) become

$$
S_{1}: p=q, \quad S_{2}: q=\sqrt{p^{2}+1},
$$

and are denoted by slow manifolds. In the limit $\epsilon=0$ it is not difficult to see that the critical points on $S_{1}$ are stable and the critical points on $S_{2}$ are stable when $p<0$ and unstable when $p>0$. When $\epsilon$ is sufficiently small and nonzero, there are boundary layers close to the slow manifolds with slow dynamics. Close to a stable part there is an attracting trajectory and close to an unstable part there is a repelling trajectory. This is the content of Tikhonov's theorem (see, e.g., [4] or (a)

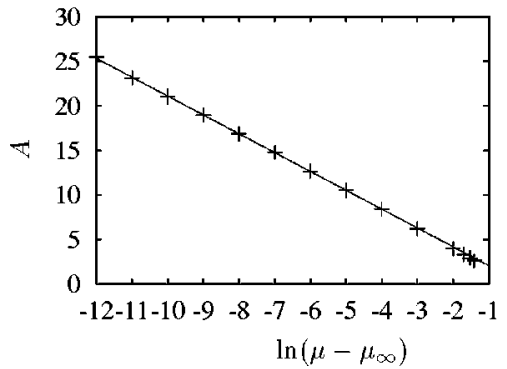

(b)

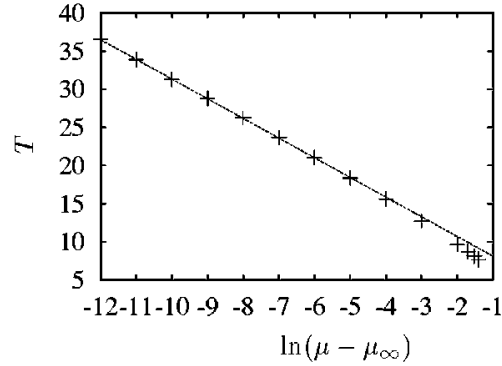

FIG. 3. Scaling of amplitude $A$ and period $T$ close to the bifurcation to infinity. The markers show numerical simulations. The lines show least-squares fit from the data with $\ln \left(\mu-\mu_{\infty}\right)$ $\leqslant-5$ yielding $A=-0.9189 \ln \left(\mu-\mu_{\infty}\right)-0.0506$, $T=-1.120 \ln \left(\mu-\mu_{\infty}\right)+5.547$. 
[12] for a geometric version based on center manifolds). The separating trajectories, denoted stable and unstable manifolds respectively, are shown for the boundary layer of $S_{2}$ in Fig. 2(a).

The explosion of the limit cycle can now be understood from the relative position of $M_{S}$ and $M_{U}$. When the configuration is as shown in Fig. 2(a), the limit cycle is attracted to $M_{S}$ on the stable side of $S_{2}$ in a slow motion. As $p=0$ is passed it is repelled up and away from $M_{U}$ and moves rapidly back towards $M_{S}$ as can be seen in (a) and (b) of the bottom row of Fig. 1. If the distance between $M_{S}$ and $M_{U}$ (measured, e.g., at $p=0$ ) is decreased, the limit cycle comes closer to $M_{U}$ and spends longer time in the unstable boundary layer before it is repelled. Hence the amplitude and period increases. If $M_{S}$ moves to the other side of $M_{U}$, the trajectories coming from the stable boundary layer enters the unstable boundary layer below $M_{U}$ for a while and are then repelled downwards into the stable boundary layer of $S_{1}$, where unbounded motion occurs. In this case no limit cycles can exist. This is shown in (c) in the bottom row of Fig. 1.

Hence, the bifurcation to infinite amplitude happens when $M_{S}$ and $M_{U}$ are the same trajectory. An asymptotic expression for this trajectory and the corresponding value of $\mu$ can be obtained as follows. Expansions of the trajectory

$$
q(p)=q_{0}(p)+\epsilon q_{1}(p)+\epsilon^{2} q_{2}(p)+\cdots,
$$

and the bifurcation point

$$
\mu_{\infty}=\mu_{0}+\epsilon \mu_{1}+\epsilon^{2} \mu_{2}+\cdots,
$$

are inserted in the Eqs. (17), and terms of the same order in $\epsilon$ are collected. To order $\epsilon^{k}$, this gives rise to an algebraic equation for $q_{k}(p)$. In general, this has a singularity at $p$ $=0$, but $\mu_{k-1}$ can be chosen in a unique way to remove the singularity and hence makes the trajectory well defined for all $p$.

Implementing this procedure in a computer algebra program yields the results in Table I, where the expansion of the parameter is shown for $\epsilon=1$. The asymptotic nature of the series is apparent, as the agreement with the numerical result

TABLE II. Asymptotic calculation of $\mu_{c}$ for Eq. (5) for $r$ $=0.005$.

\begin{tabular}{lccc}
\hline \hline $\begin{array}{l}\text { Order } \\
k\end{array}$ & $\begin{array}{c}\text { Basic term } \\
\mu_{k}^{(0)}\end{array}$ & $\begin{array}{c}\text { Correction term } \\
\mu_{k}^{(1)}\end{array}$ & $\begin{array}{c}\mu_{c} \text { for } r=0.005 \\
\sum_{n=0}^{k}\left(\mu_{k}^{(0)}+\mu_{k}^{(1)} r\right)\end{array}$ \\
\hline 0 & 1.00000000 & -1.50000000 & 0.99250000 \\
1 & -0.12500000 & 1.81250000 & 0.85843750 \\
2 & 0.03125000 & -1.23437500 & 0.88351563 \\
3 & -0.00488281 & -0.11767578 & 0.87804443 \\
4 & -0.00469971 & 0.25778198 & 0.87463364 \\
5 & 0.00757980 & -0.12494087 & 0.88158874 \\
6 & -0.00622034 & -0.04510999 & 0.87514284 \\
7 & 0.00072032 & 0.17333773 & 0.87672986 \\
8 & 0.00958744 & -0.21272633 & 0.88525366 \\
9 & -0.02425103 & 0.11999141 & 0.86160259 \\
10 & 0.03671743 & -2310.22742 & -10.6528170 \\
\hline \hline
\end{tabular}

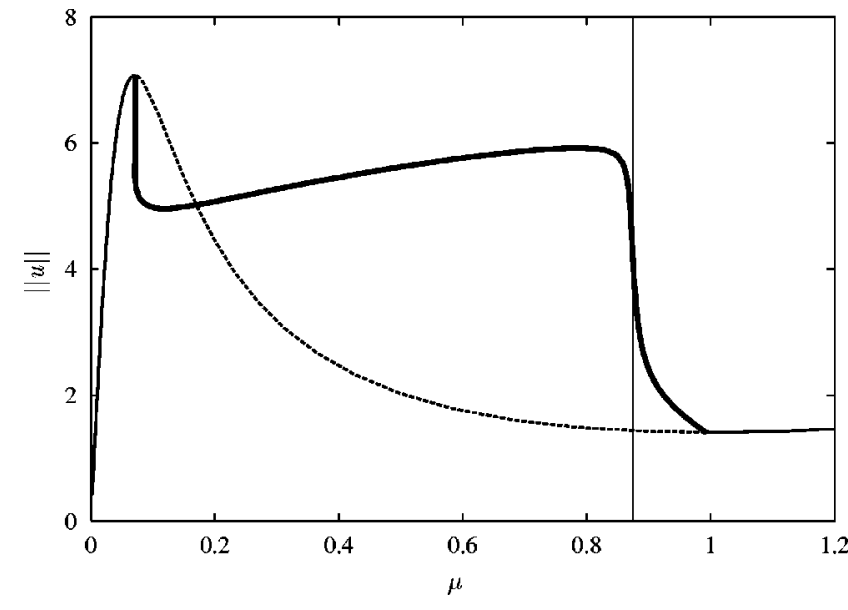

FIG. 4. Bifurcation diagram of Eq. (5) for $r=0.005$. The light line is the steady state (full line stable, dashed line unstable.) The heavy line is the limit cycle. The veritcal line is the asymptotic canard point from Table II, $\mu_{c} \approx 0.875$.

becomes poor if too many terms in the series are included. If the series for $\mu$ is truncated when the absolute value of the contribution is minimal at order 4 , the error is only about $0.4 \%$.

\section{B. Scaling}

From the geometric analysis it is also possible to understand the scaling close to the bifurcation. As the bifurcation is approached, the limit cycle spends more and more time in the unstable boundary layer of $S_{2}$. Returning to the original $(u, v)$ variables, we have here that $v \approx 1 / u$, and from the first equation of Eq. (3) we get $u(t) \approx K+\mu t$ for some constant $K$. Let $\eta=v-1 / u$ denote the deviation of the trajectory from the slow manifold. We get (a)

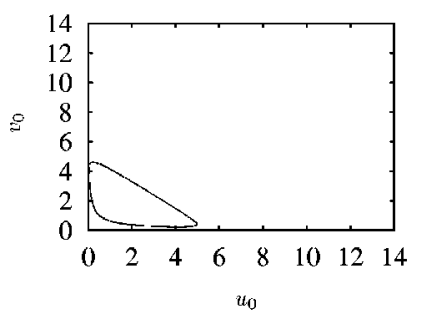

(c)

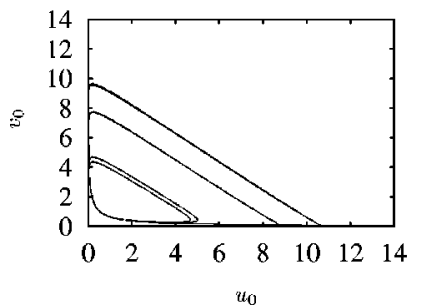

(b)

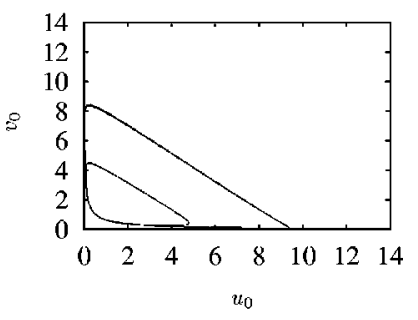

(d)

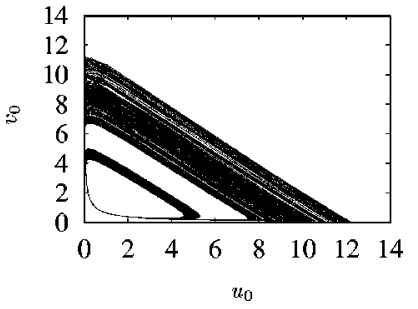

FIG. 5. Solutions of Eq. (9) showing $[u(x=0.5, t), v(x$ $=0.5, t)]$. (a) $\mu=0.904$, simple limit cycle. (b) $\mu=0.9001$, period- 2 limit cycle. (c) $\mu=0.90007$, period-4 limit cycle. (d) $\mu$ $=0.900055$, chaos . 


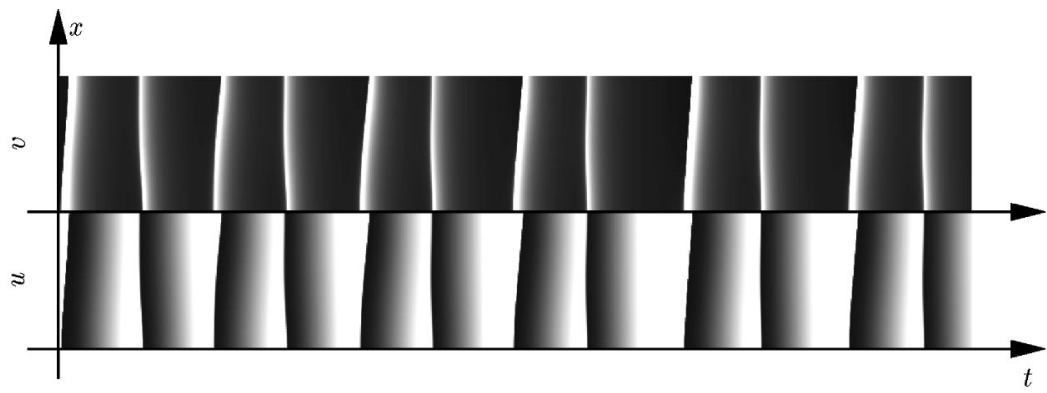

FIG. 6. Solution of Eq. (9) at $\mu=0.900055$, corresponding to Fig. 5(d). The top part shows $v$, the lower shows $u$. The concentrations are shown in a scale from black (low) to white (high).

$$
\frac{d \eta}{d t}=\eta+(K+\mu t) \eta^{2}+\frac{\mu}{(K+\mu t)^{2}} .
$$

For large $t$ the last term becomes negligible, and disregarding this term the solution of Eq. (21) becomes

$$
\eta(t)=\left(K+\mu t-\mu-\frac{e^{-t}(1+\delta K-\delta \mu)}{\delta}\right)^{-1}
$$

where $\delta=\eta(0)$. For small $\delta$, that is, for trajectories close to $S_{2}$ this is

$$
\eta(t)=\delta e^{t}+O\left(\delta^{2}\right) .
$$

Let the thickness of the boundary layer be denoted by $\Delta$. Then a trajectory leaves the boundary layer when $\eta(t)=\Delta$, that is when

$$
t=\ln \frac{\Delta}{\delta} \text { and } u=K+\mu \ln \frac{\Delta}{\delta}
$$

Assuming that the entry of the limit cycle to the unsteady boundary layer is proportional to $\mu-\mu_{\infty}$, that is, $\delta=\alpha(\mu$ $\left.-\mu_{\infty}\right)$, we get that the amplitude $A$ of the limit cycles scales as the logarithm of $\mu-\mu_{\infty}$, but in contrast to the ( $\mu$ $\left.-\mu_{\infty}\right)^{-1 / 2}$ scaling proposed in [2] on the basis of several local expansions of the limit cycle. Our result is confirmed by numerical computations shown in Fig. 3.

\section{Canard explosion}

The bifurcation to infinity is structurally unstable in the sense that a generic modification of the equations will destroy the behavior. However, an explosive change of amplitude will still occur, as we will demonstrate for the system (5). Using again the transformation (16) the system still has the form (17), now with

$$
f(u, v)=v-u v^{2}-r u, \quad g(u, v)=\mu-v .
$$

Introducing a small parameter as before, we now obtain a single slow manifold $u=v /\left(v^{2}+r\right)$, as sketched in Fig. 2(b). This is no longer unbounded in the $p$ direction, and trajectories in the boundary layer of $S_{1}$ must leave it as the turning point $T$ is reached. Here trajectories go rapidly at nearly constant $q$ to the stable part of $S_{2}$, and are not unbounded. Hence, if $M_{S}$ is below $M_{U}$ limit cycles of large but finite amplitude will occur. If $M_{S}$ is above $M_{U}$ there are smaller limit cycles that never reach $S_{1}$, and the boundary between these two kinds of limit cycles occur again exactly when $M_{S}=M_{U}$. The parameter value where this occurs has previously been denoted the canard point $\mu_{c}$ [6]. This may be determined asymptotically with the method we used before, since the procedure depends only on the system in a neighborhood of $p=0$. By this we have computed the canard point for small values of $r$ by further expanding each term of Eq. (20) in $r$ as $\mu_{k}=\mu_{k}^{(0)}+\mu_{k}^{(1)} r+\cdots$. The results of the computations are shown in Table II. Again the series is clearly asymptotic, and breaking at $k=4$ where the contribution $\mu_{k}$ is minimal we obtain $\mu_{c} \approx 0.875$. The numerically obtained bifurcation diagram in Fig. 4 confirms the interpretation of $\mu_{c}$.

\section{THE REACTION-DIFFUSION SYSTEM}

The homogeneous branch of steady solutions $[u(x, t), v(x, t)]=(1 / \mu, \mu)$ for the reaction-diffusion system (9) and (10) can loose stability in both pitchfork and Hopf bifurcations. Further, the bifurcating steady branches has secondary bifurcations, and a very rich set of possible structures exists, as described in $[9,10]$. Here we consider the complex dynamics associated with the bifurcation to infinity in a case with small diffusion coefficients (a)

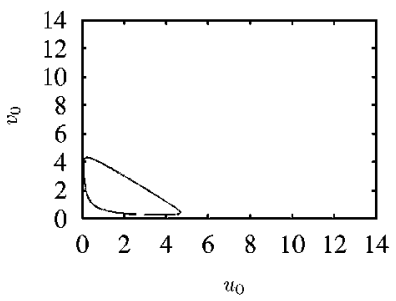

(c)

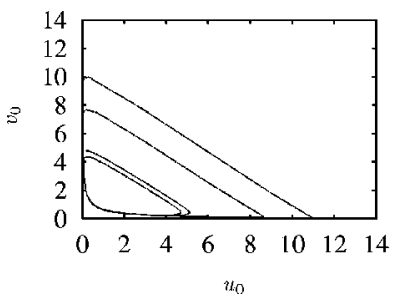

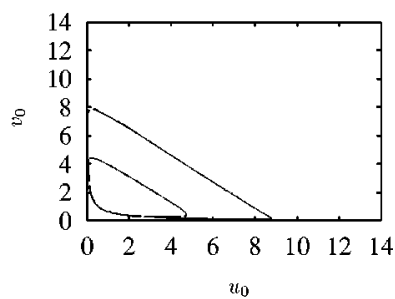

(d)

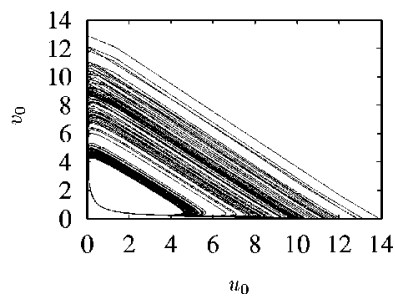

FIG. 7. Solutions of Eq. (5) with diffusion added for $r=10^{-4}$ showing $\quad[u(x=0.5, t), v(x=0.5, t)]$. (a) $\mu=0.905$. (b) $\mu$ $=0.89969$. (c) $\mu=0.89960$. (d) $\mu=0.899587$. 


$$
\lambda_{u}=\frac{0.009}{\pi^{2}}=0.0091189065, \quad \lambda_{v}=\frac{9}{10} \lambda_{u}=0.0082070159 .
$$

We have performed simulations with the program reaction diffusion system solver [13], which employs a CrankNicholson scheme with a Runge-Kutta method for the time integrations. We have used 50 spatial grid points and verified the computations by making sample runs with 100 points that yielded almost identical results. The Galerkin method used in [11] turned out to give incorrect results (negative concentrations) for the present choice of diffusion coefficients.

Results from the simulations are shown in Fig. 5. For a high value of $\mu$ a simple limit cycle exists, but as $\mu$ is decreased, period doubling bifurcations leading to chaos occur. Further decreasing $\mu$ yield chaotic transients, with a long time behavior as in Fig. 5(d), but with solutions eventually having $u \rightarrow \infty, v \rightarrow 0$.

The spatial structure of the solutions in the chaotic regime can be seen from Fig. 6, where a few oscillations are shown. Most of the time the concentrations are almost homoge- neous, and the system essentially behaves as if no diffusion was present with slow changes of concentrations. However, as $u$ becomes sufficiently large and $v$ becomes sufficiently small a fast wave moves across the domain, $u$ becomes small and $v$ becomes large, and an almost homogeneous state is reestablished. This is repeated in an irregular pattern, and the slow/fast behavior again confirms the singular perturbation nature of the system.

The unbounded solutions allowed by the reactiondiffusion system will again disappear if the reaction scheme is modified. However, the chaotic dynamics may persist, as it stems from the sensitivity of the system to the relative positions of $M_{S}$ and $M_{U}$. To demonstrate that we have performed simulations with the reaction-diffusion system where the kinetics is given by Eq. (5). Results are shown in Fig. 7, where essentially the same behavior as without the modification is found. Hence, the chaotic dynamics is not related to the bifurcation to infinity itself, but is rather associated with the sensitivity of trajectories in a parameter region where a stable and an unstable boundary layer are close and interact strongly, and may as well occur in a system that exhibits a canard explosion.
[1] J. H. Merkin, D. J. Needham, and S. K. Scott, SIAM (Soc. Ind. Appl. Math.) J. Appl. Math. 47, 1040 (1987).

[2] J. H. Merkin, D. J. Needham, and S. K. Scott, J. Eng. Math. 21, 115 (1987).

[3] B. F. Gray, M. J. Roberts, and J. H. Merkin, J. Eng. Math. 22, 267 (1988).

[4] J. Grasman, Asymptotic Methods of Relaxation Oscillations and Applications, Applied Mathematical Sciences Vol. 63 (Springer-Verlag, New York, 1987).

[5] M. Brøns and K. Bar-Eli, J. Phys. Chem. 95, 8706 (1991).

[6] M. Brøns and K. Bar-Eli, Proc. R. Soc. London, Ser. A. 445, 305 (1994).

[7] M. Brøns and J. Sturis, Int. J. Bifurcation Chaos Appl. Sci.
Eng. 2, 129 (1992).

[8] J. Sturis and M. Brøns, J. Math. Biol. 36(2), 119 (1997).

[9] J. H. Merkin and D. J. Needham, Dyn. Stab. Syst. 4, 141 (1989).

[10] D. J. Needham and J. H. Merkin, Dyn. Stab. Syst. 4, 259 (1989).

[11] R. Hill, J. H. Merkin, and D. J. Needham, J. Eng. Math. 29, 413 (1995).

[12] J. Carr, Applications of Centre Manifold Theory (Springer Verlag, New York, 1981).

[13] N. Savill and S. Mareé, RDS: Reaction Diffusion System Solver, Biomathematics group, Utrecht University, URL http:// binf.biol.ruu.nl/ $\sim$ njs/rd.html 